\title{
Correction to: Opicapone in Parkinson's disease: a profile of its
} use

\author{
Lesley J. Scott ${ }^{1}$
}

Published online: 21 November 2017

(C) Springer International Publishing AG, part of Springer Nature 2017

\section{Correction to: Drugs Ther Perspect 2017;33(7):303-10 https://doi.org/10.1007/s40267-017-0419-5}

Page 303, column 2, paragraph 1, lines 1-4: the following sentence, which previously read:

Parkinson's disease (PD), the most common chronic, progressive, neurodegenerative disease, is a manageable, but as yet, incurable disease that increases in prevalence with age $[1,2]$.

Should read:

Parkinson's disease (PD), the second most common chronic, progressive, neurodegenerative disease, is a manageable, but as yet, incurable disease that increases in prevalence with age $[1,2]$.

Page 304, column 1, paragraph 1, lines 1-4: the following text, which previously read:

levodopa and its rapid peripheral metabolism by aromatic $L$-amino acid decarboxylase to dopamine and by catechol$O$-methyltransferase (COMT) to $3 O$-methyldopa (3-OMD) [1-3].

The online version of the original article can be found under https:// doi.org/10.1007/s40267-017-0419-5.

Lesley J. Scott

dtp@adis.com

1 Springer, Private Bag 65901, Mairangi Bay, Auckland 0754, New Zealand
Should read:

levodopa and its rapid peripheral metabolism by aromatic $L$-amino acid decarboxylase to dopamine and by catechol$O$-methyltransferase (COMT) to $3 O$-methyldopa (3-OMD) $[2,3]$.

Page 306, column 2, paragraph 1, lines 1-2: the following text, which previously read:

Clinician's Global Impression of Change (PGI-C) and Patient's Global Impression of Change (PGI-C) in BIPARK I [18].

Should read:

Clinician's Global Impression of Change (CGI-C) and Patient's Global Impression of Change (PGI-C) in BIPARK I [18].

Page 308, column 2, paragraph 3, lines 1-8: the following sentence, which previously read:

Opicapone has the advantage of once-daily administration versus frequent administration with second generation COMT inhibitors, with entacapone administered with each levodopa dose (maximum of $\leq 10$ doses/day) and tolcapone administered three times daily [1-3]. Tolcapone is also associated with an increased risk of hepatotoxicity (albeit very rare), which limits its use to patients who fail to respond to other COMT inhibitors [1-3].

Should read:

Opicapone has the advantage of once-daily administration versus frequent administration with second generation COMT inhibitors, with entacapone administered with each levodopa dose (maximum of $\leq 10$ doses/day) and tolcapone administered three times daily [3]. Tolcapone is also associated with an increased risk of hepatotoxicity 
(albeit very rare), which limits its use to patients who fail to respond to other COMT inhibitors [3].

Page 309 reference list: Reference 1, which previously read:

1. Samii A, Nutt J, Ransom RR, et al. Parkinson's disease. Lancet. 2004;2004(362):1783-93
Should read:

1. Kalia LV, Lang AE. Parkinson's disease. Lancet. 2015;(386):896-912 